CrossMark

$\leftarrow$ click for updates

Cite this: Org. Biomol. Chem., 2017, 15, 555

Received 6th November 2016,

Accepted 2nd December 2016

DOI: $10.1039 / c 6 o b 02415 g$

www.rsc.org/obc

\section{Unusual reversal of enantioselectivity in the asymmetric autocatalysis of pyrimidyl alkanol triggered by chiral aromatic alkanols and amines}

\author{
Arimasa Matusmoto, a,b Satoshi Fujiwara, ${ }^{a}$ Yui Hiyoshi, ${ }^{a}$ Kerstin Zawatzky, \\ Alexey A. Makarov, ${ }^{c}{ }$ Christopher J. Welch ${ }^{c}$ and Kenso Soai*a,b
}

\begin{abstract}
Temperature dependent inversion of enantioselectivity in asymmetric catalysis is an interesting and somewhat unusual phenomenon. We have observed temperature dependent inversion of enantioselectivity in the asymmetric autocatalysis reaction when triggered by a wide scope of enantioenriched alcohols and amines. The addition reaction of diisopropylzinc to pyrimidine-5-carbaldehyde in the presence of enantiopure alcohols or amines affords the pyrimidyl alkanol product at $0{ }^{\circ} \mathrm{C}$ with high ee. However, lowering the reaction temperature to $-44{ }^{\circ} \mathrm{C}$ affords the opposite enantioselectivity.
\end{abstract}

Controlling stereochemistry is an important topic in modern organic synthesis. While accessing either enantiomer of a desired product typically requires access to both enantiomeric forms of a particular chiral catalyst or reagent, the idea of accessing either product enantiomer through a simple change in reaction conditions is quite attractive. Although various types of enantioselectivity modulation and reversal by change of solvent, additives, metals, etc. have been reported, ${ }^{1}$ enantioselectivity reversal using only temperature control is a rather rare phenomenon. ${ }^{2}$

We have studied the asymmetric autocatalysis of pyrimidyl alkanol in the addition reaction of diisopropyl zinc to the pyrimidine-5-carbaldehyde $1 .^{3}$ In this reaction, the product chiral alkanol acts as an asymmetric catalyst for its own formation, leading to autocatalytic formation of product with the same absolute configuration as the triggering alkanol, and with significant amplification of enantiomeric excess (ee). ${ }^{3 c}$ Since this autocatalytic amplification can be triggered by other materials besides the alkanol product, the reaction can function as an enantioenrichment sensor to detect various chiral environments (Scheme 1a). ${ }^{4,5}$ Hence this reaction is the sole example of a practically perfect asymmetric autocatalytic reaction with

\footnotetext{
${ }^{a}$ Department of Applied Chemistry, Tokyo University of Science, Kagurazaka, Shinjuku-ku, Tokyo 162-8601, Japan. E-mail: soai@rs.kagu.tus.ac.jp ${ }^{b}$ Research Center for Chirality, Research Institute for Science and Technology, Tokyo University of Science, Kagurazaka, Shinjuku-ku, Tokyo 162-8601, Japan ${ }^{c}$ Department of Process Research \& Development, Merck Research Laboratories, Rahway, NJ, USA
}

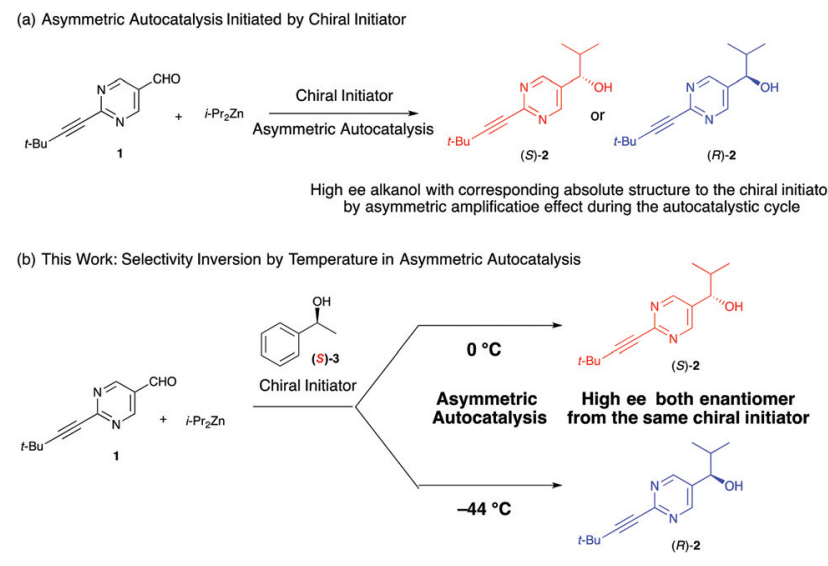

Scheme 1 Temperature dependent selectivity inversion.

amplification of asymmetry. The characteristic behaviour of this reaction has attracted much attention from diverse areas of science ${ }^{6}$ especially in the view point of origin of chirality ${ }^{7}$ and led to a number of detailed studies of reaction mechanism. ${ }^{8}$ We have previously reported an unusual selectivity inversion brought about by the presence of additives in the asymmetric autocatalysis, ${ }^{9}$ and now report the temperature induced inversion of the enantioselectivity of the asymmetric autocatalysis when triggered with enantioenriched alcohols or amines (Scheme 1b).

Asymmetric autocatalysis of the pyrimidyl alkanol system can be utilized to detect the handedness of a variety of chiral initiators, affording product with high ee even when the ee of the triggering substance is low. Although a repetition cycle of the autocatalysis reaction gives almost enantiopure alkanol, simple one step mixing of various chiral compounds with diisopropylzinc and pyrimidine-5-carabaldehyde immediately gives enantioenriched pyrimidyl alkanol with reasonably high enantiomeric excess. ${ }^{10}$ As shown in Scheme $1 \mathrm{~b}$, in the presence of (S)-1-phenethyl alcohol 3, the addition reaction of diisopropyl zinc to the pyrimidine-5-carbaldehyde affords (S)-pyrimidyl alkanol in high enantiomeric excess at $0{ }^{\circ} \mathrm{C}$ 
Table 1 Temperature dependent inversion of enantioselectivity in asymmetric autocatalysis using various chiral initiators ${ }^{d}$
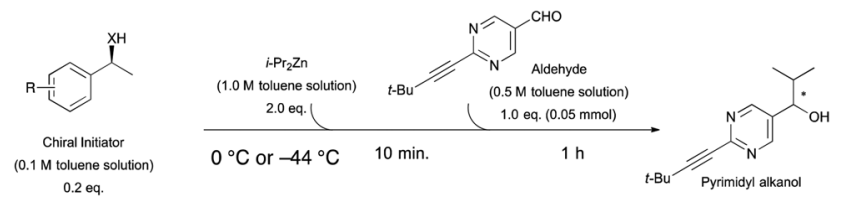

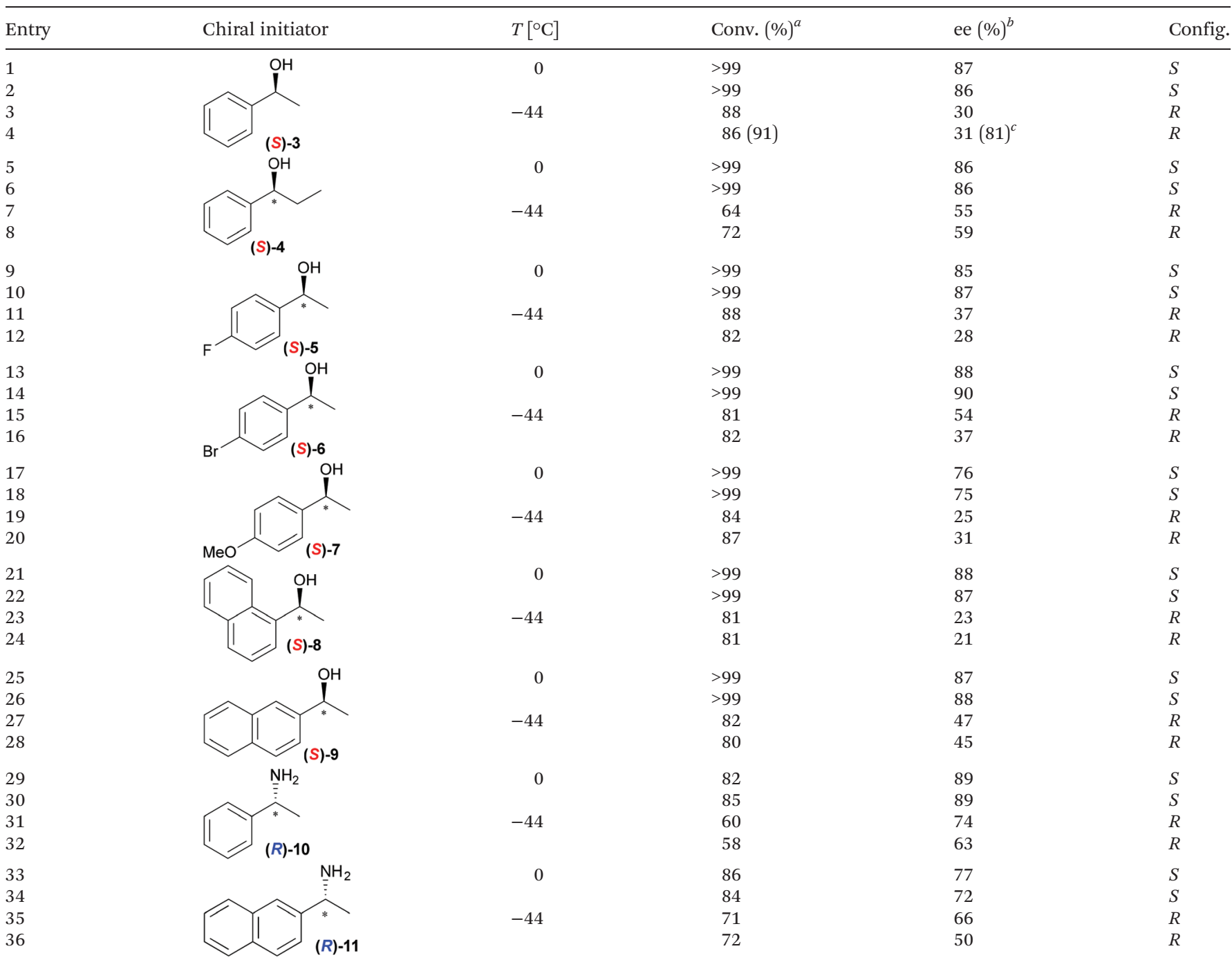

${ }^{a}$ Conversions were determined by GC using $n$-tridecane as an internal standard. ${ }^{b}$ ee was determined by SFC equipped with Chiralpak IB column. ${ }^{c}$ ee after 2 -times one-pot scale up at $-44{ }^{\circ} \mathrm{C}$ by sequential addition i- $\mathrm{Pr}_{2} \mathrm{Zn}(0.4$ and $1.6 \mathrm{mmol})$ and aldehyde $(0.2$ and $0.8 \mathrm{mmol}) .{ }^{e}$ ${ }^{d}$ Experimental detail: To a dry tube, chiral alcohol in toluene $(0.1 \mathrm{M}, 0.1 \mathrm{~mL}, 0.01 \mathrm{mmol})$ was placed and the sample tube was cooled with ice bath (for $0{ }^{\circ} \mathrm{C}$ ) or dry ice/acetonitrile bath (for $-44^{\circ} \mathrm{C}$ ). To the tube, $1 \mathrm{M}$ diisopropylzinc in toluene $(0.1 \mathrm{~mL}, 0.1 \mathrm{mmol})$ was added and stirred for $10 \mathrm{~min}$. Then 2-(3,3-dimethylbut-1-yn-1-yl)pyrimidine-5-carbaldehyde 1 in toluene $(0.5 \mathrm{M}, 0.1 \mathrm{~mL}, 0.05 \mathrm{mmol})$ was added at one time. The reaction mixture stirred for $1 \mathrm{~h}$ under the reaction temperature and quenched by the addition of $\mathrm{NH}_{4} \mathrm{Cl} / \mathrm{NH}_{4} \mathrm{OH}$ aq. The yield was determined by GC using $n$-tridecane as an internal standard and the ee was determined by SFC after short pass of $\mathrm{NH}_{2}$-silica gel. SFC conditions: CHIRALPAK® IB $(4.6 \mathrm{~mm} \phi \times 250 \mathrm{~mm}), \mathrm{MeOH} 0.3 \mathrm{~mL} \mathrm{~min}^{-1}, \mathrm{CO}_{2} 3.0 \mathrm{~mL} \mathrm{~min}^{-1}, 254 \mathrm{~nm}, 25{ }^{\circ} \mathrm{C}$, retention time (min): 1.9 for $S, 2.1$ for $R .{ }^{e}$ Experimental detail for one pot scale up: After the $1 \mathrm{~h}$ stirring of typical experimental procedure one pot scale up was performed by the addition of diisopropylzinc in toluene $(0.4 \mathrm{~mL}, 0.4 \mathrm{mmol})$ and the aldehyde 1 in toluene $n$ toluene $(0.5 \mathrm{M}, 0.4 \mathrm{~mL}, 0.2 \mathrm{mmol})$ was added at one time. After another $1 \mathrm{~h}$ stirring at the reaction temperature second time scale up was performed by the addition of diisopropylzinc in toluene $(1.6 \mathrm{~mL}, 1.6 \mathrm{mmol})$ and the aldehyde 1 in toluene $(0.5 \mathrm{M}, 1.6 \mathrm{~mL}, 0.8 \mathrm{mmol})$. The reaction was quenched and analysed after $1 \mathrm{~h}$ stirring with the same procedure.

(Table 1, entries 1 and 2). The product selectivity with this condition shows good reproducibility, however, when the same reaction is performed at $-44{ }^{\circ} \mathrm{C}$, the same $(S)$-1-phenethyl alcohol 3 shows the opposite selectivity, reproducibly giving rise to the $(R)$-alkanol (entries 3 and 4 ). Details of the relationship between temperature and the enantioselectivity of the reaction are shown in Fig. 1. A complex nonlinear relationship between temperature and ee is observed, $\dagger$ but this result 

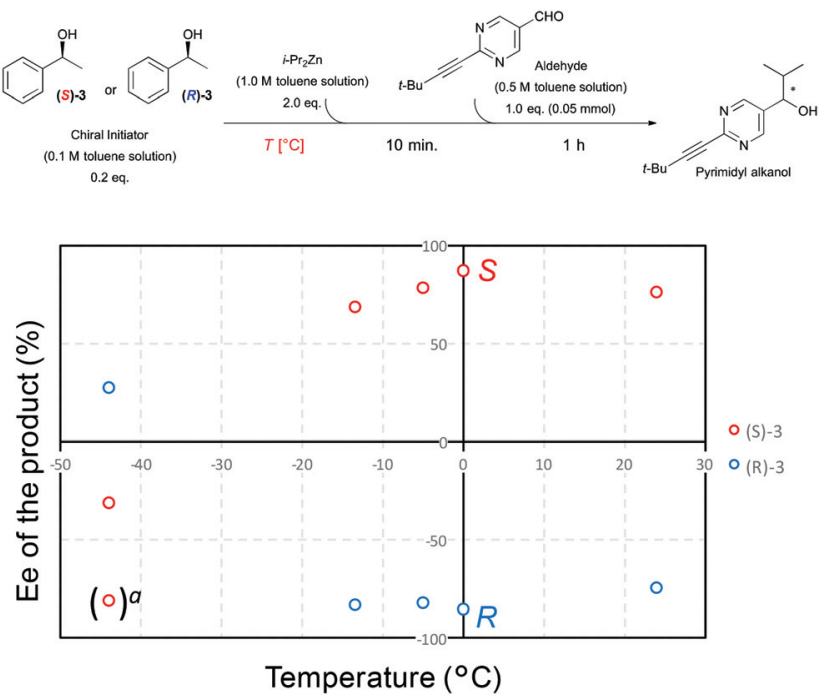

Fig. 1 Temperature dependence of the alkanol ee in the asymmetric autocatalysis initiated 1-phenethyl alcohol. ${ }^{a}$ ee after 2-times one-pot scale up of asymmetric autocatalysis at $-44^{\circ} \mathrm{C}$.

clearly highlights the inversion in enantioselectivity at a temperature between 0 to $-44{ }^{\circ} \mathrm{C}$, with temperature dependent reversal of enantioselectivity also being observed with $(R)-1$-phenethy alcohol triggered reaction. Although, the ee of the obtained alkanol at $-44{ }^{\circ} \mathrm{C}$ was slightly lower compared to that of the $0{ }^{\circ} \mathrm{C}$, the conversion was still good and the amplification of ee occurs to give the high ee of alkanol after one-pot further asymmetric autocatalysis with scale up even at this low temperature (entry 4 , in bracket).

Temperature dependent inversion of enantioselectivity is also observed with reaction triggering by the other chiral secondary alcohols (Table 1). In addition to 1-phenethyl alcohol 3, 1-phenyl-1-propanol 4 also causes selectivity inversion. Various chiral $(S)$-alcohols with aromatic substitution on the 1-position such as fluorophenyl 5, bromophenyl 6, methoxyphenyl 7, 1- and 2-naphtyl 8, 9 all induce $(S)$-alkanol formation in the asymmetric autocatalysis at $0{ }^{\circ} \mathrm{C}$. However, all these chiral alcohols induce the formation of the opposite $(R)$-alkanol product at $-44{ }^{\circ} \mathrm{C}$. It should be noted that all chiral secondary alcohols with aryl and methyl substitution examined in the study show the same selectivity and temperature inversion behaviour. In addition to the alcohols, amines also induced temperature dependent inversion of selectivity. For example, triggering with $(R)$-1-phenylethan-1-amine 10 gives rise to the $(S)$-alkanol at $0{ }^{\circ} \mathrm{C}$, but affords the $(R)$-alkanol product at $-44{ }^{\circ} \mathrm{C}$. Similarly, triggering with $(R)-1$-(naphthalen2-yl)ethan-1-amine $\mathbf{1 1}$ also led to enantioselectivity inversion between $0{ }^{\circ} \mathrm{C}$ and $-44{ }^{\circ} \mathrm{C}$. Interestingly, although the selectivity trend of amines (heterochiral product produced at $0{ }^{\circ} \mathrm{C}$ ) was different from that of the alcohols 3-9 (homochiral product produced at $0^{\circ} \mathrm{C}$ ) the reversal of enantioselectivity at $-44^{\circ} \mathrm{C}$ was seen in all cases.

The detailed mechanism underlying this interesting temperature-dependent inversion in enantioselectivity is not yet clear. Temperature induced reversal of selectivity is sometimes explained by a change in the sign of free energy brought about by the entropic contributions to selectivity overwhelming the enthalpic contributions as temperature changes. ${ }^{1,2}$ In this asymmetric autocatalysis system, zinc alkoxide is also known to form various oligomeric species, ${ }^{8}$ and temperaturedependent changes in the aggregation state of the zinc alkoxide complex, or its association with the triggering agent, might also be involved for this selectivity inversion. While a comprehensive explanation awaits further studies, it should be noted that temperature dependent inversion of enantioselectivity is possible with this well-known enantioselective autocatalytic system.

In conclusion, we have presented several examples of the temperature dependent inversion of chiral initiation in an asymmetric autocatalysis reaction. Although the mechanistic details of this inversion are not yet clear, selectivity inversion by temperature was observed with a variety of different chiral alcohols and amines. These results offer intriguing clues for improved mechanistic understanding of analyte triggered asymmetric autocatalysis. Furthermore, this result provides an important reminder that the temperature effect in asymmetric additions of organozinc reagents is sometimes not so simple, with low temperature reactions not always leading to the best results.

We thank the Grant-in-aid for Scientific Research (JSPS KAKENHI) and the MEXT-Supported Program for the Strategic Research Foundation at Private Universities, 2012-2016 and the MRL Postdoctoral Research Fellows Program for a fellowship (K. Z.).

\section{Notes and references}

$\dagger$ The reaction under much lower temperature such as $-78^{\circ} \mathrm{C}$ is very sluggish and almost all used aldehyde recovered in this conditions.

1 (a) B. H. Buschmann, H. Scharf, N. Hoffmann and P. Esser, Angew. Chem., Int. Ed. Engl., 1991, 30, 477-515; (b) J. Escorihuela, M. I. Burguete and S. V. Luis, Chem. Soc. Rev., 2013, 42, 5595-5617.

2 (a) Y. Inoue, T. Yokoyama, N. Yamasaki and A. Tai, J. Am. Chem. Soc., 1989, 111, 6480-6482; (b) J. Otera, K. Sakamoto, T. Tsukamoto and A. Orita, Tetrahedron Lett., 1998, 39, 3201-3204; (c) M. P. Sibi, U. Gorikunti and M. Liu, Tetrahedron, 2002, 58, 8357-8363; (d) C. P. Casey, S. C. Martins and M. A. Fagan, J. Am. Chem. Soc., 2004, 126, 5585-5592; (e) V. S. Chan, M. Chiu, R. G. Bergman and F. D. Toste, J. Am. Chem. Soc., 2009, 131, 6021-6032; (f) P. Pongrácz, T. Papp, L. Kollár and T. Kégl, Organometallics, 2014, 33, 1389-1396; $(g)$ G. Storch and O. Trapp, Angew. Chem., Int. Ed., 2015, 54, 3580-3586; (h) I. Méndez, R. Rodríguez, V. Polo, V. Passarelli, F. J. Lahoz, P. García-Orduña and D. Carmona, Chem. Eur. J., 2016, 22, 11064-11083.

3 (a) K. Soai, T. Shibata, H. Morioka and K. Choji, Nature, 1995, 378, 767-768; (b) T. Shibata, S. Yonekubo and 
K. Soai, Angew. Chem., Int. Ed., 1999, 38, 659-661; (c) I. Sato, H. Urabe, S. Ishiguro, T. Shibata and K. Soai, Angew. Chem., Int. Ed., 2003, 42, 315-317.

4 Example of asymmetric autocatralysis. (a) I. Sato, R. Sugie, Y. Matsueda, Y. Furumura and K. Soai, Angew. Chem., Int. Ed., 2004, 43, 4490-4492; (b) T. Kawasaki, M. Sato, S. Ishiguro, T. Saito, Y. Morishita, I. Sato, H. Nishino, Y. Inoue and K. Soai, J. Am. Chem. Soc., 2005, 127, 32743275; (c) T. Kawasaki, K. Suzuki, Y. Hakoda and K. Soai, Angew. Chem., Int. Ed., 2008, 47, 496-499; (d) T. Kawasaki, Y. Matsumura, T. Tsutsumi, K. Suzuki, M. Ito and K. Soai, Science, 2009, 324, 492-495; (e) A. Matsumoto, H. Ozaki, S. Harada, K. Tada, T. Ayugase, H. Ozawa, T. Kawasaki and K. Soai, Angew. Chem., Int. Ed., 2016, 55, 15246-15249.

5 (a) K. Soai and T. Kawasaki, in Topics in Current Chemistry, ed. K. Soai, Springer Berlin Heidelberg, Berlin, Heidelberg, 2008, vol. 284, pp. 1-31; (b) T. Kawasaki and K. Soai, Bull. Chem. Soc. Jpn., 2011, 84, 879-892; (c) T. Kawasaki and K. Soai, Isr. J. Chem., 2012, 52, 582-590; (d) T. Kawasaki, I. Sato, H. Mineki, A. Matsumoto and K. Soai, J. Synth. Org. Chem., Jpn., 2013, 71, 109-123; (e) K. Soai, T. Kawasaki and A. Matsumoto, Acc. Chem. Res., 2014, 47, 3643-3654; (f) K. Soai, T. Kawasaki and A. Matsumoto, Chem. Rec., 2014, 70-83.

6 (a) M. Avalos, R. Babiano, P. Cintas, J. L. Jiménez and J. C. Palacios, Chem. Commun., 2000, 887-892; (b) D. G. Blackmond, Proc. Natl. Acad. Sci. U. S. A., 2004, 101, 5732-5736; (c) J. Podlech and T. Gehring, Angew. Chem., Int. Ed., 2005, 44, 5776-5777; (d) J. M. Brown, I. D. Gridnev and J. Klankermayer, Amplification of Chirality, in Topics in Current Chmistry, ed. K. Soai, 2008, vol. 284, pp. 35-65; (e) T. Gehring, M. Busch, M. Schlageter and D. Weingand, Chirality, 2010, 22(Suppl 1), E173-E182; $(f)$ G. Lente, Symmetry, 2010, 2, 767-798; $(g)$ B. Barabás, J. Tóth and G. Pályi, J. Math. Chem., 2010, 48, 457-489; (h) A. J. Bissette and S. P. Fletcher, Angew. Chem., Int. Ed., 2013, 52, 12800-12826; (i) O. Fülöp and B. Barabás, J. Math. Chem., 2016, 54, 10-17.

7 (a) B. L. Feringa and R. A. van Delden, Angew. Chem., 1999, 111, 3624-3645; (b) M. M. Green, J.-W. Park, T. Sato,
A. Teramoto, S. Lifson, R. L. B. Selinger and J. V. Selinger, Angew. Chem., Int. Ed., 1999, 38, 3138-3154; (c) J. M. Ribó, J. Crusats, F. Sagués, J. Claret and R. Rubires, Science, 2001, 292, 2063-2066; (d) D. K. Kondepudi and K. Asakura, Acc. Chem. Res., 2001, 34, 946-954; (e) H. Zepik, E. Shavit, M. Tang, T. R. Jensen, K. Kjaer, G. Bolbach, L. Leiserowitz, I. Weissbuch and M. Lahav, Science, 2002, 295, 1266-1269; (f) K. Mislow, Collect. Czech. Chem. Commun., 2003, 68, 849-864; $(g)$ S. Pizzarello and A. L. Weber, Science, 2004, 303, 1151; (h) C. Viedma, Phys. Rev. Lett., 2005, 94, 65504; (i) R. Breslow and M. S. Levine, Proc. Natl. Acad. Sci. U. S. A., 2006, 103, 12979-12980; (j) V. A. Soloshonok, H. Ueki, M. Yasumoto, S. Mekala, J. S. Hirschi and D. A. Singleton, J. Am. Chem. Soc., 2007, 129, 12112-12113; (k) I. Weissbuch and M. Lahav, Chem. Rev., 2011, 111, 3236-3267; (l) Y. Saito and H. Hyuga, Rev. Mod. Phys., 2013, 85, 603-621; ( $m$ ) S. Olsson, P. M. Björemark, T. Kokoli, J. Sundberg, A. Lennartson, C. J. McKenzie and M. Håkansson, Chem. - Eur. J., 2015, 21, 5211-5219.

8 (a) I. Sato, D. Omiya, H. Igarashi, K. Kato, Y. Ogi, K. Tsukiyama and K. Soai, Tetrahedron: Asymmetry, 2003, 14, 975-979; (b) G. Ercolani and L. Schiaffino, J. Org. Chem., 2011, 76, 2619-2626; (c) T. Gehring, M. Quaranta, B. Odell, D. G. Blackmond and J. M. Brown, Angew. Chem., Int. Ed., 2012, 51, 9539-9542; (d) I. D. Gridnev and A. K. Vorobiev, ACS Catal., 2012, 2, 2137-2149; (e) A. Matsumoto, T. Abe, A. Hara, T. Tobita, T. Sasagawa, T. Kawasaki and K. Soai, Angew. Chem., Int. Ed., 2015, 54, 15218-15221; (f) A. Matsumoto, S. Fujiwara, T. Abe, A. Hara, T. Tobita, T. Sasagawa, T. Kawasaki and K. Soai, Bull. Chem. Soc. Jpn., 2016, 89, 1170-1177.

9 (a) F. Lutz, T. Igarashi, T. Kawasaki and K. Soai, J. Am. Chem. Soc., 2005, 127, 12206-12207; (b) D. Lavabre, J. C. Micheau, J. R. Islas and T. Buhse, J. Phys. Chem. A, 2007, 111, 281-286; (c) F. Lutz, T. Igarashi, T. Kinoshita, M. Asahina, K. Tsukiyama, T. Kawasaki and K. Soai, J. Am. Chem. Soc., 2008, 130, 2956-2958.

10 C. J. Welch, K. Zawatzky, A. A. Makarov, S. Fujiwara, A. Matsumoto and K. Soai, Org. Biomol. Chem., 2016, 11, DOI: 10.1039/C6OB01939K. 